\title{
Ultra-low Noise EEG at LSBB: New results
}

\author{
Nazanin Hamzei ${ }^{1}$, Zoya Bastany ${ }^{1}$, Catherine R. Jutzeler ${ }^{2}$, Matt Yedlin ${ }^{1}$, John L.K. Kramer ${ }^{3}$, \\ John D. Steeves ${ }^{3}$, and Guy A. Dumont ${ }^{1, a}$ \\ ${ }^{1}$ Department of Electrical and Computer Engineering, The University of British Columbia, \\ Vancouver, BC, V6T 1Z4, Canada \\ ${ }^{2}$ Spinal Cord Injury Center, University Hospital Balgrist, University of Zurich, Zurich \\ ${ }^{3}$ International Collaboration On Repair Discoveries (ICORD), Blusson Spinal Cord Centre, 818 West \\ 10th Avenue, Vancouver, BC, V5Z 1M9, Canada
}

\begin{abstract}
In this study, we investigate functional correlates of gamma band oscillations in low-noise EEG signals acquired in the LSBB shielded capsule and compare them to signals acquired in a typical hospital environment. Using a research-grade EEG acquisition system, we acquired 64-channel EEG recordings from three volunteers performing several cognitive, sensory, and motor tasks in both LSBB and hospital settings. Time-frequency analysis on the signals acquired in both environments reveals that the task-induced increase in gamma band $(>30 \mathrm{~Hz})$ energy relative to the resting state EEG is more prominent in signals acquired at LSBB, suggesting that task-specific changes in EEG are better reflected and more readily detected in signals acquired at LSBB. These results further demonstrate the potential value of low-noise settings such as the LSBB for conducting challenging highfrequency EEG studies.
\end{abstract}

\section{Introduction}

The electroencephalogram (EEG) has proven to be a useful information source in the analysis of brain activity, neural plasticity, and the diagnosis of various neurological disorders, as well as the development of intelligent brain-computer interfaces (BCIs). Advancements in EEG signal acquisition and analysis have enabled improved diagnostic capabilities for seizure detection [1], sleep disorders [2], and Alzheimer's disease [3], just to name a few. EEG has also been extensively used to monitor anesthesia and sedation in the operating room and intensive care unit [4].

EEG can be defined as a representation of neuronal activity (e.g. summated extracellular action potential frequencies) within different brain regions and is usually acquired noninvasively from the surface of the scalp. To reduce the disruptive effects of electrical interference and movement (i.e. noise), depending on the neuronal activity of interest, EEG is often band-pass filtered at different frequencies from 0.5 to $100 \mathrm{~Hz}$ and is then studied in terms of activity across different frequency bands; including $\delta(0-4 \mathrm{~Hz}), \theta(4-7 \mathrm{~Hz}), \alpha$ $(8-12 \mathrm{~Hz}), \beta(12-30 \mathrm{~Hz})$, and $\gamma(30-100 \mathrm{~Hz})$. Clinically, EEG frequencies are most often studied from 0.5 to $30 \mathrm{~Hz}$ with the gamma $(\gamma)$ band being ignored. The gamma band is ignored due to the use of filters to suppress unwanted electromagnetic noise from powered $(50$ or $60 \mathrm{~Hz}$ ) electrical outlets. In recent years however, the gamma band has received greater

${ }^{a}$ Corresponding author: guyd@ece.ubc.ca

(C) The Authors, published by EDP Sciences. This is an Open Access article distributed under the terms of the Creative Commons Attribution License 4.0 (http://creativecommons.org/licenses/by/4.0/). 


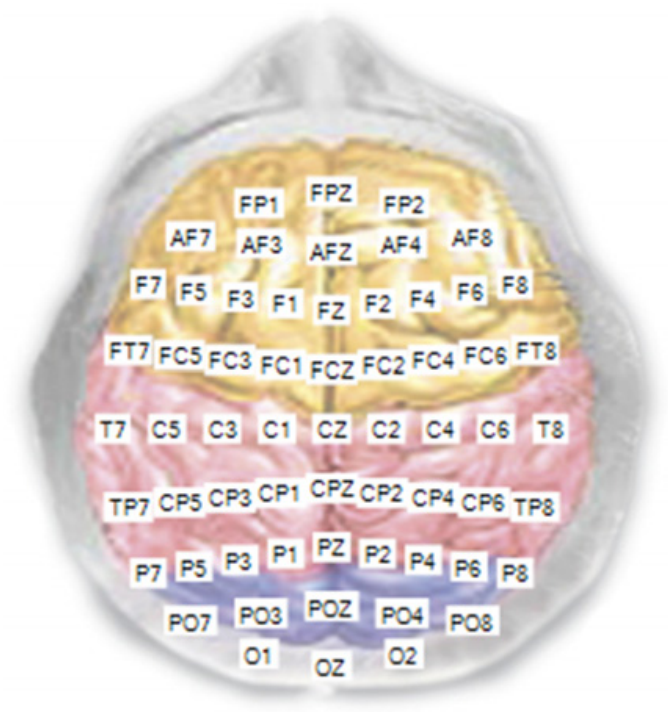

Figure 1. 10-10 electrode montage used for EEG acquisition. Reference and ground electrodes are not shown in the figure.

scrutiny and is typically known to underlie and be modulated by sensorimotor behaviors and internal cognitive processes such as working memory and attention [10].

In this study, we further investigate the potential of the ultra-shielded capsule at the Laboratoire Souterrain Bas Bruit (LSBB in Rustrel, Pays d'Apt, France) for acquisition of clean EEG signals, with a focus on analysis of high frequencies (gamma-band) in search for novel activity patterns. In a previous study, we have shown that EEG signals acquired in LSBB are free from electromagnetic interference and can be acquired and analyzed without the need for notch filtering with battery-powered EEG equipment $[6,7]$. This facilitates examination of gamma-band EEG signals acquired in the LSBB while searching for functionally significant high-frequency EEG correlates which would otherwise be obscured by common filters of high-frequency electromagnetic noise. Our objective in this study is to examine the functionally relevant increase in gamma band energy from continuous EEG recordings acquired at LSBB, and test whether gamma-band EEG is accentuated and more readily detected in a low-noise environment than in a typical hospital setting.

\section{Data acquisition}

The battery-operated NR SIGN EEG 5000Q 64-channel EEG system (NR SIGN Inc., New Westminster, BC, Canada) was used for non-invasive EEG recording (Fig. 1). Data was acquired at a sampling rate of $500 \mathrm{~Hz}$ with 16-bit resolution, and was transferred using a USB cable to a laptop computer (also running on battery power) through the NR SIGN EEG application software. The equipment was used to acquire raw, unfiltered continuous EEG recordings at LSBB from seven subjects. Similar recordings were then acquired in ICORD (International Collaboration On Repair Discoveries), a hospital facility within Vancouver General Hospital, using the same equipment and protocols to allow for comparison between the two recording environments. Five subjects had volunteered for data acquisition at ICORD, four of whom were also present during acquisition in LSBB. For the purpose of the study, three subjects were selected out of the four subjects common to both environments, since the 
acquired data for one subject was found to be corrupted with large movement and muscle artifacts, as well as EOG (high-amplitude electro-oculogram artifact caused by blinking of the eyes) to a high extent.

In each of the environments, the subjects were asked to perform a variety of cognitive, sensory, and motor tasks while EEG was acquired in real time. First, EEG data was collected from the subjects in a darkened room during a relaxed (resting) state as a baseline condition (with either eyes open or eyes closed). The subjects were then asked to perform specific tasks over a 30 -second period, while resting for 30 seconds in between the test intervals. The tasks included: 1) counting backwards by 7 from a large randomly selected number; 2) performing an increasingly challenging "matching" memory task on an iPad; 3) performing reciprocal dorsal and plantar flexion movements of the right ankle; and 4) performing repeated flexionextension of the right wrist. All subjects were right-hand dominant. To assess the effects of pain on the EEG, the subjects were subjected to 5) "brushing" of the adductor pollicis region of the right thumb with a cotton swab as an innocuous tactile stimulation task; and 6) application of a hot pack (recorded skin temperature $=34-36^{\circ} \mathrm{C}$ ) to the same adductor pollicis region as a noxious stimulus, while rating their pain perception using the $(0-10,10$ being most painful) visual analog scale (VAS). For the latter task, only two out of the three subjects were included in the analysis since one subject did not continue the recording at the hospital environment, claiming the stimulus was too hot to tolerate.

\section{Data analysis}

\subsection{Pre-processing}

All recordings were visually inspected in the time domain for removal of periods containing broadly distributed, high-amplitude movement artifacts, electrode saturation, and other irregular artifacts. Electrodes with poor skin contact, judged by their abnormal activity patterns throughout the length of the recording, were removed from the data at each of the recordings and the analysis was based on stable channel recordings. Occurrence of eye blinks and eye movements were not criteria for data rejection since their frequency content is usually below $15 \mathrm{~Hz}$ and did not overlap with the gamma band frequencies. Naturally, there was no need for application of notch filters to recordings acquired in LSBB. As for recordings at ICORD, no notch filer was used either owing to the fact that the batteryoperated acquisition system was also able to minimize external electromagnetic power-line interference automatically using well-calibrated differential amplifiers.

\subsection{Time-frequency analysis}

In order to quantitatively measure the oscillatory activity of EEG signals, time-frequency spectrograms were computed using the Stockwell transform [8]. Also known as the S-transform, the Stockwell transform is a variant of the continuous wavelet transform (CWT) which uses a moving and scalable localizing Gaussian window. While having the advantage of frequency-dependent resolution over Short-time Fourier Transform (STFT), the S-transform is also shown to have a frequency invariant amplitude response, contrary to the Continuous Wavelet Transform which forces more attenuation on high frequencies. These features make the S-transform an ideal tool for detection of high-frequency activity in EEG signals. The $\mathrm{S}$-transform of a signal $x(t)$ is given by:

$$
S(\tau, f)=\int_{-\infty}^{+\infty} x(t)|f| \frac{e^{\frac{(t-\tau)^{2} f^{2}}{2}}}{\sqrt{2 \pi}} e^{-2 \pi i f t} d t
$$



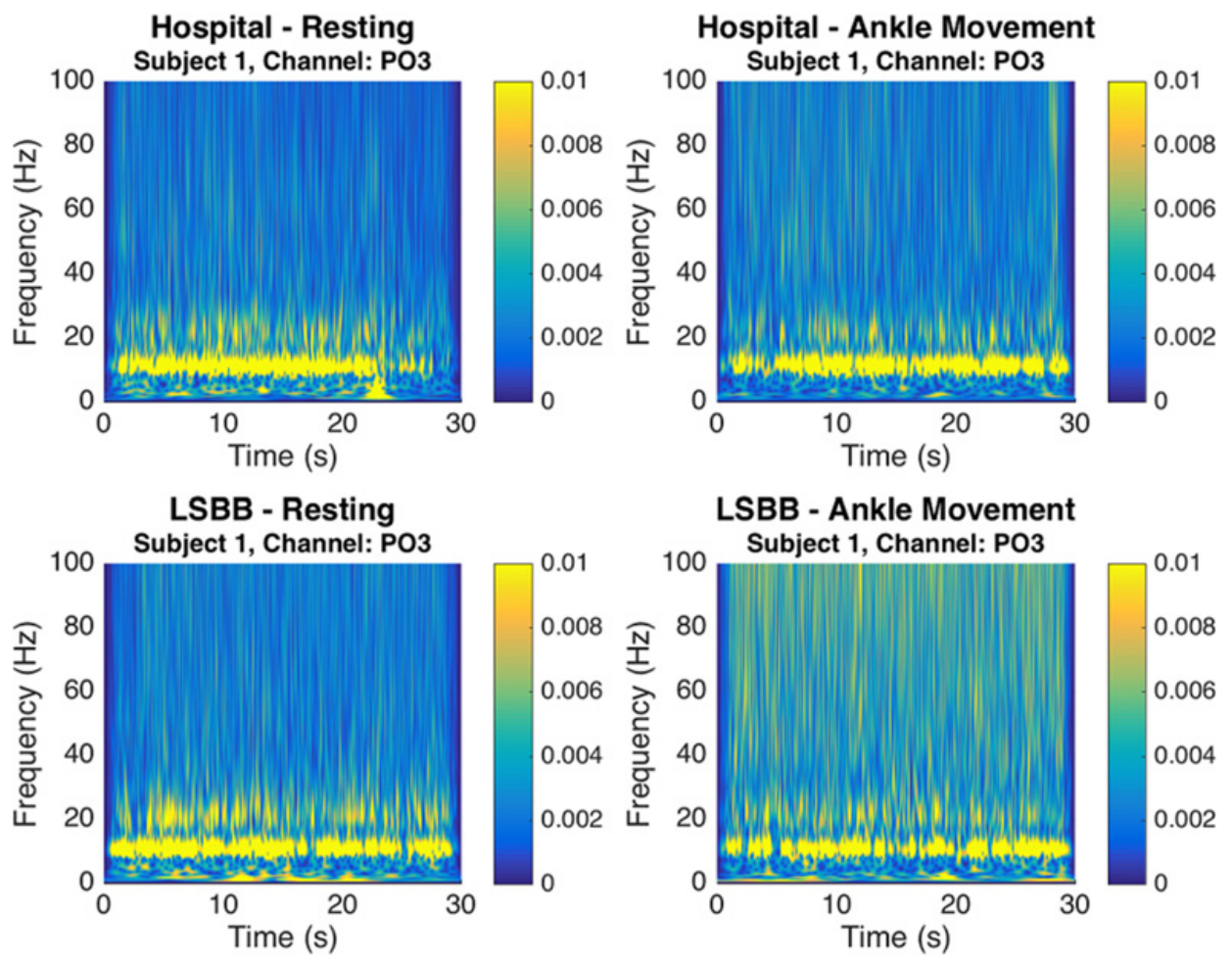

Figure 2. Magnitude of the Stockwell transform of normalized EEG signals from one channel (PO3) of a subject during rest (left column) and ankle movement (right column), acquired at the hospital facility (top row) versus the LSBB (bottom row). Task-specific changes within gamma band are more significant and easier to detect in LSBB.

Figure 2 shows the magnitude of the S-transformed EEG signals of one subject during 30-second intervals of rest and a repetitive motor activity (ankle movement) in the different recording environments. Comparing the figures on the left (resting state) with those on the right (motor task), we observe that the motor activity has caused power increases within the gamma band $(\mathrm{f}>30 \mathrm{~Hz}$ ); however, this increase in high-frequency power is more apparent in the LSBB when compared to the hospital environment.

To quantitatively assess the task-specific changes in the gamma band EEG, we calculated the task-rest gamma band energy ratios of the $\mathrm{S}$ transform and compared them between LSBB and the hospital environment. To this end, artifact-free recordings for all subjects and all tasks were first segmented into consecutive epochs of 30 seconds. Each epoch was then normalized in order to reduce the influence of electrode impedance and alleviate the disparities across subjects due to factors such as skin conductivity, which yielded epochs with comparable amplitude values. Channel-wise normalization was performed in the time domain by removing the mean and dividing by the root-mean-square value of the corresponding channel [4], according to the equation below:

$$
x_{N}(t)=\frac{x(t)-\bar{x}}{x_{r m s}}
$$


Table 1. Grouping of electrodes based on different sites of the brain.

\begin{tabular}{|l|l|}
\hline Brain Region & Electrodes \\
\hline Prefrontal & FPZ, FP1, FP2, AFZ, AF3, AF4, AF7, AF8 \\
\hline Frontal & FZ, F1, F2, F3, F4, F5, F6, F7, F8, FCZ, FC1, FC2, FC3, FC4 \\
\hline Central & CZ, C1, C2, C3, C4, CPZ, CP1, CP2, CP3, CP4 \\
\hline Left Temporal & FC5, FT7, C5, T7, CP5, TP7 \\
\hline Right Temporal & FC6, FT8, C6, T8, CP6, TP8 \\
\hline Left Parietal & P3, P5, P7, PO3, PO7 \\
\hline Right Parietal & P4, P6, P8, PO4, PO8 \\
\hline Parietal & PZ, P1, P2, POZ \\
\hline Occipital & OZ, O1, O2 \\
\hline
\end{tabular}

We measure the high frequency activity during each of the epochs by computing the total gamma band energy of the epoch in the time-frequency space, defined as:

$$
E_{\gamma}=\int_{f_{L}=30}^{f_{H}=100} \int_{\tau_{i}=0}^{\tau_{f}=30}\left|S_{N}(\tau, f)\right|^{2} d \tau d f .
$$

Where $\left|S_{N}(\tau, f)\right|$ refers to the magnitude of the $\mathrm{S}$ transform of the normalized signal (Eq. (2)), and integration is performed over the entire epoch length (30 seconds) and within the gamma band $(30-100 \mathrm{~Hz})$. Consequently, responses to each task for each subject were expressed as task-rest ratios; that is, given there are $N$ epochs corresponding to the rest condition and $M$ epochs belonging to a particular task, we compute a total of $M * N$ ratios by dividing the total gamma band energy of each task epoch by the total gamma band energy of each of the resting-state epochs. We then compare the entire set of ratios (including all subjects) at nine functionally different brain regions (see Table 1) in LSBB with that of the hospital environment.

\section{Discussion}

The results from time-frequency analysis across all subjects in both environments are summarized in Fig. 3, where each sub-plot corresponds to a specific grouping of electrodes (brain regions) according to Table 1. We have grouped the tasks into three categories: cognitive tasks (Fig. 3a) including counting and matching, motor tasks (Fig. 3b) including right ankle movement and right wrist movement, and right hand sensory tasks (Fig. 3c) including brushing and application of a noxious heat pack to the dorsal surface of the right thumb. Tasks belonging to each category are shown alongside each other. For each task, we have shown a boxplot of the task-rest ratios acquired in the hospital environment (left) along with a boxplot of the ratios acquired at LSBB. Shown in the plots are the median value, as well as the $25 \%$ and $75 \%$ quartiles, and the whiskers representing $\pm 2.7 \sigma$ or 99.3 percent coverage given the data is normally distributed. These plots allow for comparison of the distribution of gamma band task-rest ratios in LSBB and hospital across different brain regions.

A quick look at Fig. $3 \mathrm{~b}$ reveals that for both of the motor tasks, the gamma band motorrest ratios are significantly larger and more readily detected in LSBB across all regions of the brain. While the median of motor-rest ratios at LSBB is always greater than one, the median of the ratios at the hospital stays close to one, suggesting that the motor tasks were not readily detectable at the hospital. This is clearly an advantage for studies in a low electromagnetic noise environment like the LSBB, indicating its potential for detection of EEG benchmarks related to understanding central nervous system (CNS) control of basic motor tasks or early detection of disorders associated with changes in motor behaviors (e.g. Epilepsy, Parkinson's disease, etc.). Low-noise environments, like the LSBB, would also 

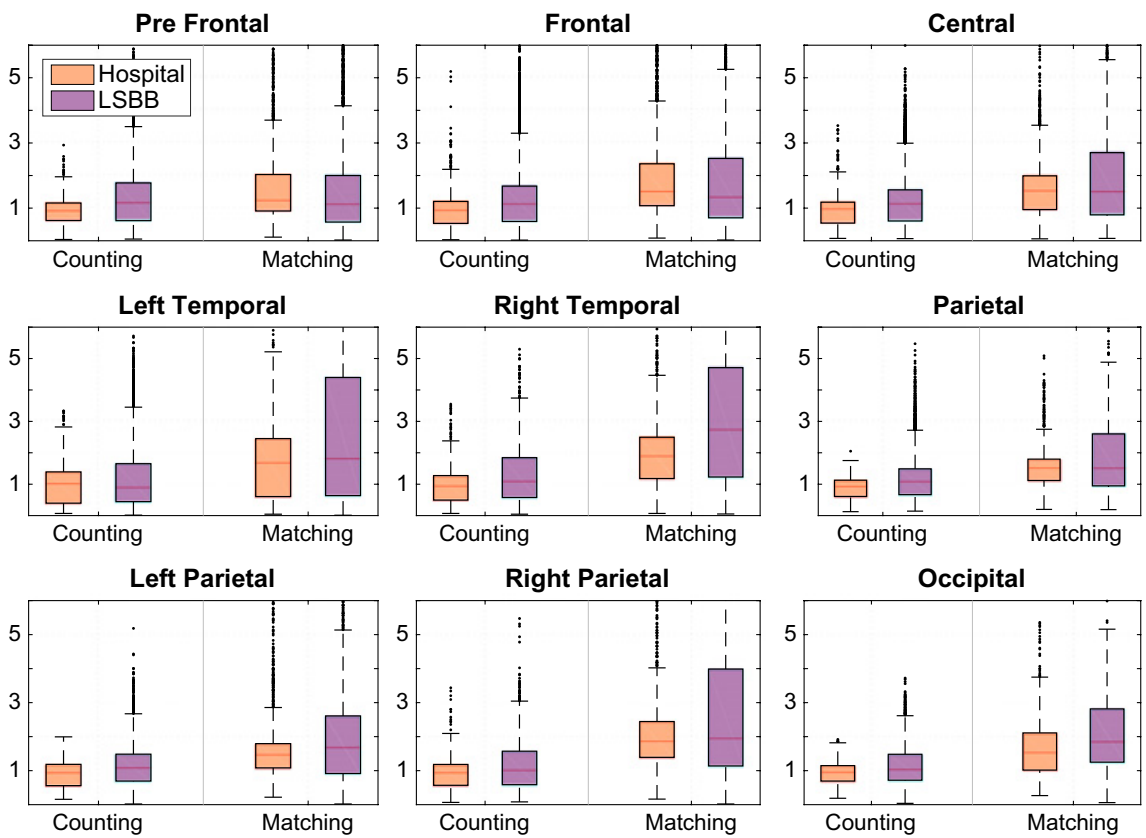

(a)
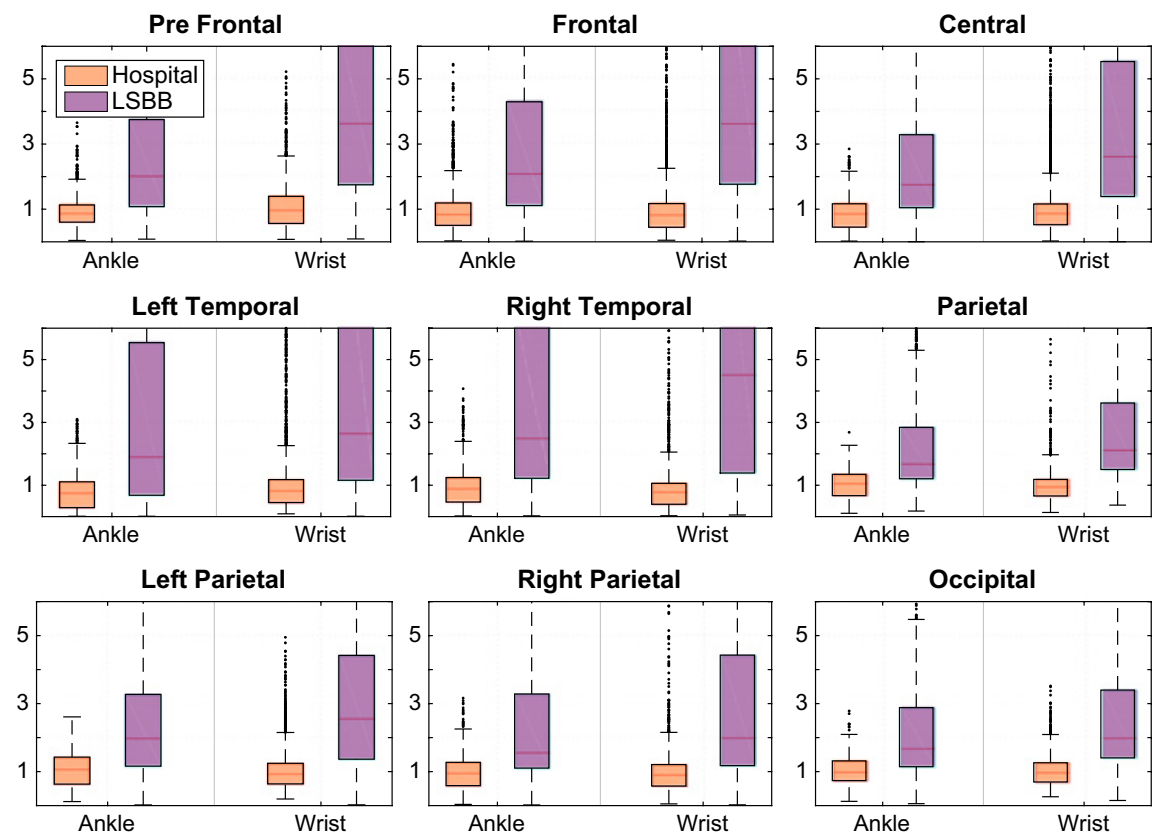

(b)

Figure 3. Comparison of distribution of task-rest ratios between signals acquired at LSBB and the hospital at different brain regions based on the grouping scheme outlined in Table 1. a) Cognitive tasks including counting and matching, b) motor tasks including ankle and wrist movements, c) sensory tasks including brushing and application of heat pack. 


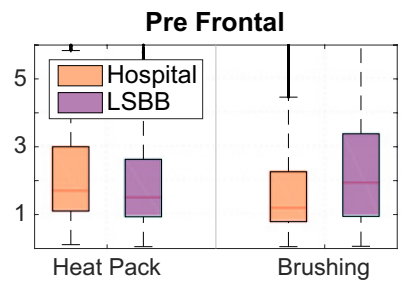

Left Temporal

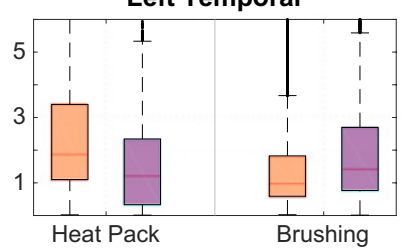

Left Parietal

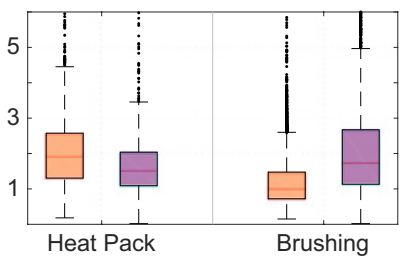

Frontal

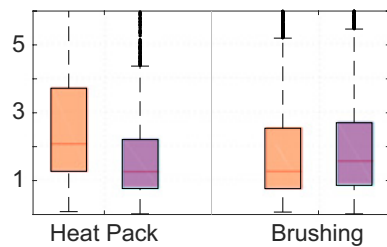

Right Temporal

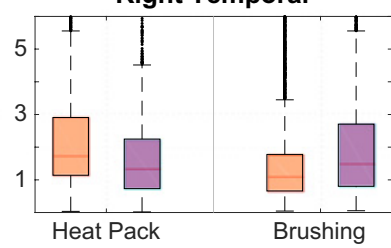

Right Parietal

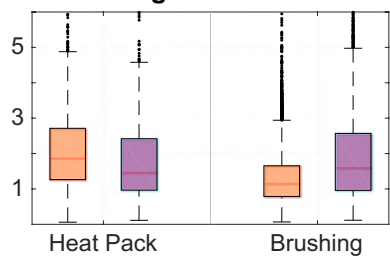

Central

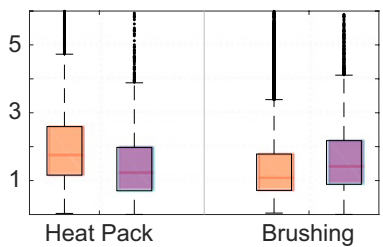

Parietal

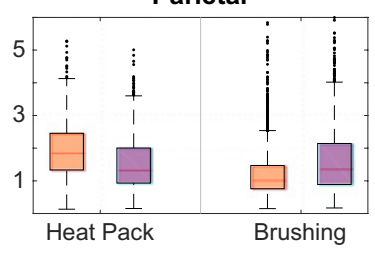

Occipital

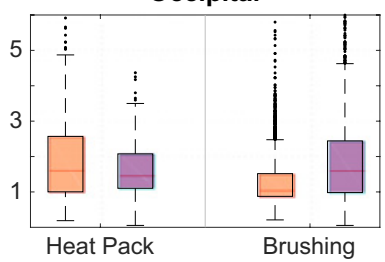

(c)

Figure 3. Continued.

facilitate a greater understanding for potential subtle changes in cortical plasticity after CNS motor injuries (e.g. spinal cord or brain damage).

We have depicted the ratios for cognitive tasks in Fig. 3a. Although having slightly higher values in LSBB, backward counting-rest ratios are mainly distributed around one in both environments, suggesting that gamma band may not be significantly involved in continuous counting tasks. We have found this to be the case also in a previous study [6]. On the other hand, matching-rest ratios are mainly greater than one in both environments, and their values are higher in LSBB at temporal and occipital regions of the brain. As an explanation for why a global increase in ratios is not seen at LSBB, it could be claimed that the increase in gamma band due to matching is more focused, contrary to the motor functions which exhibit a more global cortical response. Thus, in brain regions where the gamma-band content is boosted due to the cognitive activity associated with the matching task, the ratio increase is more prominent within the low-noise environment of the LSBB than the hospital environment. This hypothesis is backed by the fact that temporal lobes are known to be associated with visual memory and object recognition [9], two functions that are specifically needed for a matching task.

Brushing-rest and noxious heat-rest ratios were also compared between the two environments (Fig. 3c). This figure demonstrates that the median of brushing-rest ratios is slightly higher in LSBB compared to the hospital, thus revealing the potential of LSBB in identifying the brushing-induced high frequency activity. However, the heat-rest ratios are lower in LSBB than in the hospital environment. Explaining this issue, it is worth mentioning that the average of the VAS pain ratings across all recordings and all subjects was 5.5 at the 
hospital and 1.5 at LSBB, suggesting that despite the efforts to keep the experiment conditions equal, the subjects experienced more pain in the hospital facility than in LSBB. We might therefore hypothesize that the lower ratios in LSBB are caused by the lower pain experience in comparison with ICORD, which would in turn yield lower gamma band activity in LSBB as the strength of gamma band oscillations has been shown to correlate well with the intensity of the perceived pain [10]. However, since only a small number of subjects (two) were included for the heat task, caution must be exercised in interpreting the results pertaining to this task.

\section{Conclusion}

We have summarized a small pilot study on the potential of an ambient low-noise environment (e.g. LSBB) for the easy identification of task-specific high-frequency gamma-band EEG signals. Our results demonstrate that functionally correlated gamma-band EEG patterns can be better detected in low-noise conditions when compared with a typical hospital environment. This motivates the design of more informative studies with the end goal of defining potentially novel and predictive high-frequency EEG (gamma-band benchmarks) for a better understanding of central neuronal function and CNS disorders.

To address the limitations of the current work, studies using a larger number of subjects are necessary for more stringent statistical validation and more confident conclusions. One might also consider designing a protocol for detection of event-related potentials (ERPs) with time-locked experimental trials as opposed to spontaneous EEG, in order to more thoroughly investigate the role of gamma-band EEG activity in task-specific sensorimotor behaviors and/or cognitive activities.

\section{References}

[1] T.N. Alotaiby, S.A. Alshebeili, T. Alshawi, I. Ahmad, F.E. Abd El-Samie, EEG seizure detection and prediction algorithms: a survey, EURASIP Journal on Advances in Signal Processing 2014, 1-21 (2014).

[2] S. Kubicki, W. Scheuler, H. Wittenbecher, Short-term sleep EEG recordings after partial sleep deprivation as a routine procedure in order to uncover epileptic phenomena: an evaluation of 719 EEG recordings, Epilepsy Res. Suppl., 2, 217-230 (1991).

[3] J. Jeong, EEG dynamics in patients with Alzheimer's disease, Clinical neurophysiology, 115, 1490-1505 (2004).

[4] T. Zikov, S. Bibian, G.A. Dumont, M. Huzmezan, C.R. Ries, Quantifying cortical activity during general anesthesia using wavelet analysis, IEEE Transactions on Biomedical Engineering, 53, 617-632 (2006).

[5] M.W. Howard, D.S. Rizzuto, J.B. Caplan, J.R. Madsen, J. Lisman, R. AschenbrennerScheibe, A. Schulze-Bonhage, M.J. Kahana, Gamma oscillations correlate with working memory load in humans, Cerebral cortex 13, 1369-1374 (2003).

[6] A.S. Zandi, G.A. Dumont, M.J. Yedlin, P. Lapeyrie, C. Sudre, S. Gaffet, Scalp EEG Acquisition in a Low-Noise Environment: A Quantitative Assessment, IEEE Transactions on Biomedical Engineering, 58, 2407-2417 (2011).

[7] A.S. Zandi, G.A. Dumont, M.J. Yedlin, P. Lapeyrie, C. Sudre, S. Gaffet, Analysis of scalp EEG recorded in a low-noise environment, EDP Sciences (2011).

[8] R.G. Stockwell, L. Mansinha, R.P. Lowe, Localization of the complex spectrum: the $S$ transform, IEEE Transactions on Signal Processing, 44, 998-1001 (1996). 
[9] N. Axmacher, D.P. Schmitz, T. Wagner, C.E. Elger, J. Fell, Interactions between medial temporal lobe, prefrontal cortex, and inferior temporal regions during visual working memory: a combined intracranial EEG and functional magnetic resonance imaging study, The Journal of Neuroscience 28, 7304-7312 (2008).

[10] Z.G. Zhang, L. Hu, Y.S. Hung, A. Mouraux, G.D. Iannetti, Gamma-band oscillations in the primary somatosensory cortex - a direct and obligatory correlate of subjective pain intensity, The Journal of Neuroscience 32, 7429-7438 (2012). 